\title{
Potential of a Cyclone Prototype Spacer to Improve In Vitro Dry Powder Delivery
}

\author{
Irene Parisini $•$ Sean J. Cheng $•$ Digby D. Symons $•$ Darragh Murnane
}

Received: 28 June 2013 / Accepted: 14 October 2013 / Published online: 15 November 2013

(C) The Author(s) 2013. This article is published with open access at Springerlink.com

\begin{abstract}
Purpose Low inspiratory force in patients with lung disease is associated with poor deagglomeration and high throat deposition when using dry powder inhalers (DPIs). The potential of two reverse flow cyclone prototypes as spacers for commercial carrier-based DPIs was investigated.

Methods Cyclohaler ${ }^{\circledR}$, Accuhaler ${ }^{\circledR}$ and Easyhaler ${ }^{\circledR}$ were tested with and without the spacers between 30 and $60 \mathrm{Lmin}^{-1}$. Deposition of particles in the next generation impactor and within the devices was determined by high performance liquid chromatography.

Results Reduced induction port deposition of the emitted particles from the cyclones was observed due to the high retention of the drug within the spacers (e.g. salbutamol sulphate (SS): $67.89 \pm 6.51 \%$ at $30 \mathrm{Lmin}^{-1}$ in Cheng 1). Fine particle fractions of aerosol as emitted from the cyclones were substantially higher than the DPIs alone. Moreover, the aerodynamic diameters of particles emitted from the cyclones were halved compared to the DPIs alone (e.g. SS from the Cyclohaler ${ }^{\circledR}$ at 4 kPa: $1.08 \pm 0.05 \mu \mathrm{m}$ vs. $3.00 \pm 0.12 \mu \mathrm{m}$, with and without Cheng 2, respectively) and unaltered with increased flow rates.
\end{abstract}

Conclusion This work has shown the potential of employing a cyclone spacer for commercial carrier-based DPIs to improve inhaled drug delivery.

KEY WORDS cyclone $\cdot$ dry powder inhalers · fine particle fraction · oropharyngeal deposition $\cdot$ spacers

I. Parisini $\cdot$ D. Murnane $(\bowtie)$

Department of Pharmacy, University of Hertfordshire

College Lane, Hatfield ALI 0 9AB, UK

e-mail:d.murnane@herts.ac.uk

S. J. Cheng • D. D. Symons

Department of Engineering, University of Cambridge

Cambridge CB2 IPZ, UK

\section{INTRODUCTION}

Passive dry powder inhalers (DPIs) have developed a strong place in the therapy of pulmonary diseases over the last 25 years, even representing the dominant inhaled therapy in some markets (1). One of the main advantages of DPIs compared to metered dose inhalers (MDIs) is surely the avoidance of a requirement for actuation-inhalation coordination by the user (2). Nevertheless, passive DPIs pose some major challenges as a universal therapy of lung diseases. The de-aggregation of the formulation into an aerosol suitable for inhalation is dependent on the interaction between a powder formulation and the airflow which is drawn through the device (3-5). Many patients with obstructive lung disease are unable to generate an aerosol cloud through high resistance devices (6). In traditional products, even with formulations optimized for in vitro drug delivery, the in vivo efficiency (lung deposition) of DPIs has been reported to be low and to be variable (7). The degree of throat deposition has been identified as a major cause of variability in efficiency of marketed DPIs and MDIs (8).

The success of inhalation therapy depends on the complex interaction between a powder formulation and the airflow drawn through a DPI. In most cases the device design is optimized to maximize aerosolization of a specific formulation using in vitro testing. The majority of DPIs (9) contain a blend of micronized drug particles blended with non-respirable carrier particles. Micronized particles which are not removed from the carrier upon inhalation are unable to reach the lung, depositing instead within the oropharynx (5). Oropharyngeal deposition is well understood with MDIs, where large droplets with high velocity deposit in the throat-one of the leading causes of side-effects in inhalation therapy (10). In order to avoid such problems with MDIs, the use of spacer chamber is recommended to reduce the velocity and droplet size of the aerosol ultimately inhaled by the patient; thereby reducing the oropharyngeal deposition (11). 
The magnitude of the fine particle (respirable) dose emitted from DPIs has been clearly demonstrated to depend on the airflow rate through many commercial devices $(4,6)$. Several clinical studies report variability in the magnitude of inspiratory flows generated by patients when using DPIs $(6,12,13)$ and many patients with lung disease struggle to inhale with sufficient strength through medium-to-high resistance devices. The result is high variability in the liberation of fine (inhalable) particles from marketed DPIs that were well characterized using in vitro testing $(14,15)$. Oropharyngeal deposition is high for DPIs (16, 17) due to the force of the inhalation manoeuvre being insufficient to deaggregate drug/carrier mixtures effectively. Low-to-medium resistance devices appear an effective option for chronic obstructive pulmonary disease patients to achieve the high inhalation flow rates required for effective deagglomeration (18). Conversely, the inhalation of particles at high velocities from DPIs promotes inertial impaction of inhaled particles within the oropharynx (19).

There is clearly a challenge to address the oropharyngeal deposition of DPIs, as well as minimize the variability between patients in the aerodynamic diameter and respirable fractions arising from flow rate variability. Studies from the late 1990s (20, 21) showed potential to reduce oropharyngeal deposition using spacer devices for DPIs, similarly to with MDIs. A more recent CFD-led study (computational fluid dynamics) developed a spacer that improved aerosol penetration past the oropharynx for Astra Zeneca's Turbuhaler ${ }^{\text {TM }}$ (22). In addition, spacer use at low flow rates was also shown to reduce the fraction of large particles reaching the oropharynx (23). The promise of such early findings to translate into commercial or clinical success is puzzling. However, it may be due to the specificity of the prototypes for specific devices, and also because the properties of the aerosol emitted from the DPI itself into the spacer remain susceptible to the inter-patient variability in the flow rate.

In recent years, miniature cyclone technology has been developed for DPIs to capture the particles with high momentum liberating only the fine particles for inhalation (e.g. the Airmax ${ }^{\mathrm{TM}}$ ) with improved consistency of delivered dose at various flow rates (24). The Conix ${ }^{\text {TM }}$ device family incorporating reverse-flow cyclones, developed by Cambridge Consultants Ltd. (25) and licensed by 3 M Drug Delivery Systems (3 M Ltd) (26), effectively retain large agglomerates inside the device. The technology employs a cyclone chamber (cone section in Fig. 1) where an axially downward-flowing vortex is formed upon inhalation. A secondary axially upward-flowing vortex towards the exit orifice is formed at the core due to the changing diameter of the conical region. Particles of large mass and inertia are retained in the cone, and only the finest particles (e.g. $<5 \mu \mathrm{m}$ ) can escape the cyclone for inhalation (26). Reverse-flow cyclones offer potential to function as a DPI spacer to screen out the non-respirable particles in aerosols emitted from conventional DPIs. However for equivalency to the marketed product, passage through the

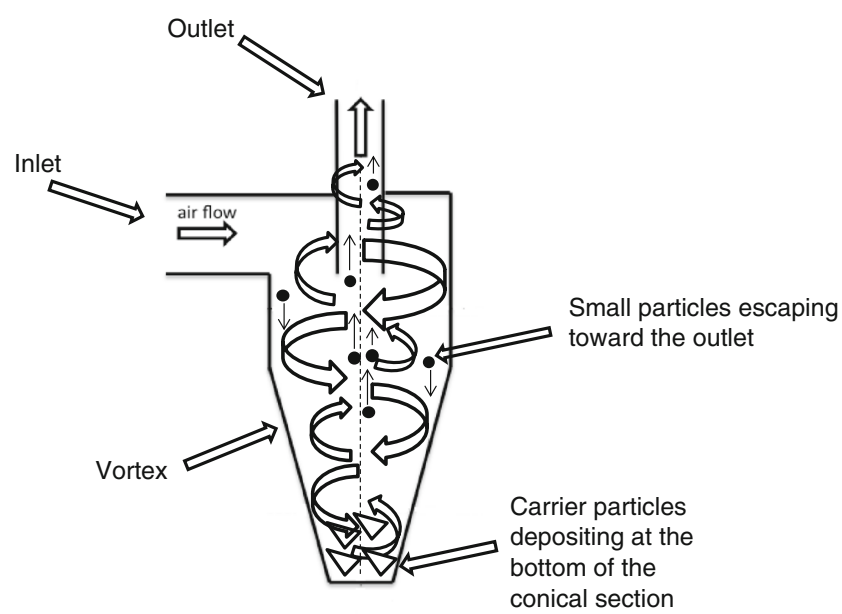

Fig. I The reverse flow cyclone principle.

cyclone should not lead to further aerosol deagglomeration. CFD investigations of miniature cyclones spacers $(27,28)$ indicated the particle size output to be restricted to respirable particles. As a result cyclone spacers with generic applicability to lung drug delivery have been prototyped. The aim of the current work was to investigate in vitro drug delivery for a range of marketed inhalers with the prototypes to investigate minimization of throat deposition from carrier-based DPIs.

\section{MATERIALS AND METHODS}

\section{Materials}

Micronized salbutamol sulphate (SS) was purchased from GlaxoSmithKline Research and Development (Ware, UK). Micronized fluticasone propionate (FP) was purchased from Sicor de Mexico (Toluca, Mexico) and micronized budesonide (Bud) was supplied from LGM Pharma (Boca Raton, USA). Salmeterol xinafoate (SX) was purchased from Vamsi Labs Ltd (Solapur, India). Lactose monohydrate, chromatography grade methanol, hydrochloric acid solution (HCl) 5M, n-Hexane were purchased from FisherScientific (Loughborough, UK). Hipersolv ${ }^{\circledR}$ grade ammonium acetate was purchased from Lab3 Ltd (Northampton, UK). A Luna $3 \mu \mathrm{m}$ C18 column $(150 \mathrm{~mm} \times 4.6 \mathrm{~mm} \times 3 \mu \mathrm{m})$ was obtained from Phenomenex (Macclesfield, UK). Silicone oil-Dow Corning Corporation 200® fluid was obtained from Sigma-Aldrich (Gillingham, UK). The DPIs tested were an Easyhaler/Budesonide ${ }^{\mathrm{TM}}$ $400 \mu \mathrm{g} /$ dose (purchased from Orion Pharma, Newbury, UK), a Seretide/Accuhaler TM $50 \mu \mathrm{g} / 500 \mu \mathrm{g} /$ dose and Ventolin/ Accuhaler TM $200 \mu \mathrm{g} /$ dose (purchased from Allen \& Hanburys, Uxbridge, Middlesex, UK) and a Cyclohaler ${ }^{\circledR}$ device (obtained from AAH Hospital Supplies, Coventry, UK). Size 4 hard gelatine capsules were obtained from Meadow Laboratories Ltd. (Romford, UK). Cyclone spacers were manufactured in- 
house (University of Cambridge, UK) from Perspex blocks (Engineering \& Design Plastics Ltd, Cambridge, UK); the exit duct was cut and polished from stock brass tubing (Engineering Department Storeroom, University of Cambridge, UK). The flow meter (model number DMF2), next generation impactor (NGI) and a model HCP5 vacuum pump were all purchased from Copley Scientific Ltd (Nottingham, UK).

\section{Cyclone Manufacture}

Two miniature reverse-flow cyclones (Cheng 1 and Cheng 2) were manufactured to have a theoretical particle cut-off diameter below $5 \mu \mathrm{m}$ for flow rates between 30 and $120 \mathrm{Lmin}^{-1}$ (with reference to empirical models of cyclone performance (29)). The first cyclone (hereafter referred to as Cheng 1) was machined from Perspex with a circular inlet orifice of diameter $4.1 \mathrm{~mm}$ to facilitate relatively high inlet velocities with high pressure drops. The second cyclone (hereafter: Cheng 2) with a $6 \times 10 \mathrm{~mm}$ rectangular slot inlet was then created with lower relative pressure drops and inlet velocities. For dimensional accuracy, easy sample collection and interchange ability both geometries were manufactured in three separate sections: exit duct (top), inlet and cylinder body (mid) and cone (bottom). The bottom of the cone section had a through-hole for sample collection, which was sealed during experiments. In Fig. 2 the dimensions of both cyclones are reported. An inlet adapter was also manufactured out of Perspex to enable connectivity with commercial DPI devices (Fig. 3).

\section{Quantitative Analysis by High Performance Liquid Chromatography (HPLC)}

The HPLC instrument used was an Agilent 1050 Series (Agilent Technologies UK Ltd., Edinburgh, UK). The stationary phase was a Luna $3 \mu \mathrm{m} \mathrm{C18}$ column ( $150 \mathrm{~mm} \times 4.6 \mathrm{~mm} \times 3 \mu \mathrm{m})$. The mobile phase was filtered through a $0.2 \mu \mathrm{m}$ nylon filter and

\begin{tabular}{c|c|c} 
& Cheng 1 & Cheng 2 \\
\hline $\mathrm{D}$ & 20 & 21 \\
$\mathrm{D}_{\mathrm{e}}$ & 5.9 & 5.9 \\
$\mathrm{a}$ & $\varnothing 4.1^{1}$ & 10.1 \\
$\mathrm{~b}$ & - & 6 \\
$\mathrm{H}$ & 57.9 & 56.4 \\
$\mathrm{~h}$ & 24.7 & 23.5 \\
$\mathrm{~S}$ & 17.3 & 23.5 \\
$\mathrm{~B}$ & 6.2 & 7.1
\end{tabular}

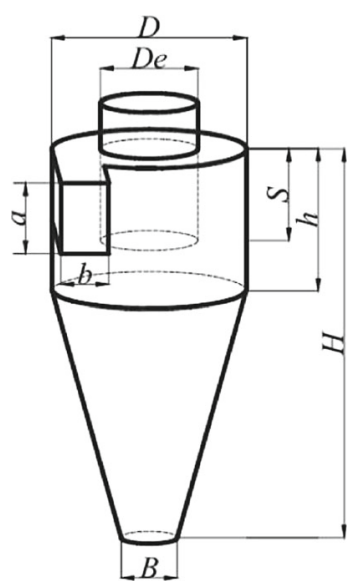

Fig. 2 Dimensions of simulated cyclone geometries (units in $\mathrm{mm}$ ). 'Cheng I was manufactured with a circular inlet orifice, diameter $4.1 \mathrm{~mm}$. degassed by ultrasonication for $30 \mathrm{~min}$. To analyze Bud the mobile phase used was a 75:25 (v/v) mixture of methanol: $0.6 \%$ (w/v) aqueous ammonium acetate. The column temperature was set at $40^{\circ} \mathrm{C}$ with UV detection at $240 \mathrm{~nm}$. To analyze SX and FP the mobile phase used was the same as per Bud. The column temperature was set at $40^{\circ} \mathrm{C}$ with $\mathrm{UV}$ detection at $228 \mathrm{~nm}$. To analyze SS the mobile phase used was an 80:20 $(\mathrm{v} / \mathrm{v})$ mixture of $0.6 \%(\mathrm{w} / \mathrm{v})$ aqueous ammonium acetate: methanol and $\mathrm{pH}$ was adjusted to 4.5 with $\mathrm{HCl} 1 \mathrm{M}$. The column temperature was set at $50^{\circ} \mathrm{C}$ with $\mathrm{UV}$ detection at $272 \mathrm{~nm}$. For all the methods the flow rate was set at $1.00 \mathrm{ml} \mathrm{min}{ }^{-1}$, with injection volume of $50 \mu \mathrm{l}$. To validate the methods, the concentration range used was between 0.5 and $50 \mu \mathrm{g} / \mathrm{ml}$ for Bud, SX and FP, and $1-50 \mu \mathrm{g} / \mathrm{ml}$ for SS.

\section{Cyclohaler ${ }^{\circledR}$ Capsule Filling}

The mixture of micronized SS and lactose was removed from marketed Ventolin/Accuhaler ${ }^{\mathrm{TM}}$ products. Size 4 hard gelatine capsules (Meadow Laboratories Ltd., Romford, UK) were accurately hand-filled with approximately $0.0125 \mathrm{~g}$ of the blend.

\section{Aerosolization Assessment Using Next Generation Impactor for Cheng I}

A solution of $1 \%$ of silicon oil in n-Hexane was prepared as coating agent for the impactor plates of the NGI. Approximately $15 \mathrm{ml}$ of the solution was added into the first stage, rotated and transferred into the second stage and so on until the last stage was covered. The plates were left under the fume hood in order to allow the hexane to evaporate leaving a thin layer of silicon as coating.

Aerosolization studies were performed using the NGI with a model HCP5 vacuum pump. The NGI was assembled according to the requirements of the British Pharmacopeia. The flow rates used were 30, 45, and $60 \mathrm{Lmin}^{-1}$ (with each actuation lasting 8.0, 5.3 and $4.0 \mathrm{~s}$, respectively). The mixture of micronized SS and lactose was removed from marketed Ventolin/Accuhaler ${ }^{\mathrm{TM}}$ products. The Ventolin ${ }^{\mathrm{TM}}$ formulation was aerosolized using a Cyclohaler ${ }^{\circledR}$ device with and without the Cheng $1.15 \mathrm{ml}$ of mobile phase for SS was added in the central cup of the pre-separator and the flow rate was adjusted with the aid of a flow meter. Drug was recovered from the device, capsules and NGI apparatus as follows. The Cyclohaler ${ }^{\circledR}$ and the capsules were washed with $20 \mathrm{ml}$ of mobile phase and transferred into a volumetric flask. The bottom of the cyclone, the mouthpiece and the throat were each washed with $50 \mathrm{ml}$ of mobile phase; whilst the upper section of the cyclone was washed with $10 \mathrm{ml}$. For collecting the drug from the pre-separator $100 \mathrm{ml}$ of mobile phase was used for washing and dissolution of deposited drug. The volumetric flasks were sonicated using a sonicator (Kerry, Germany) for 2 min. When the Cyclohaler ${ }^{\circledR}$ was employed 


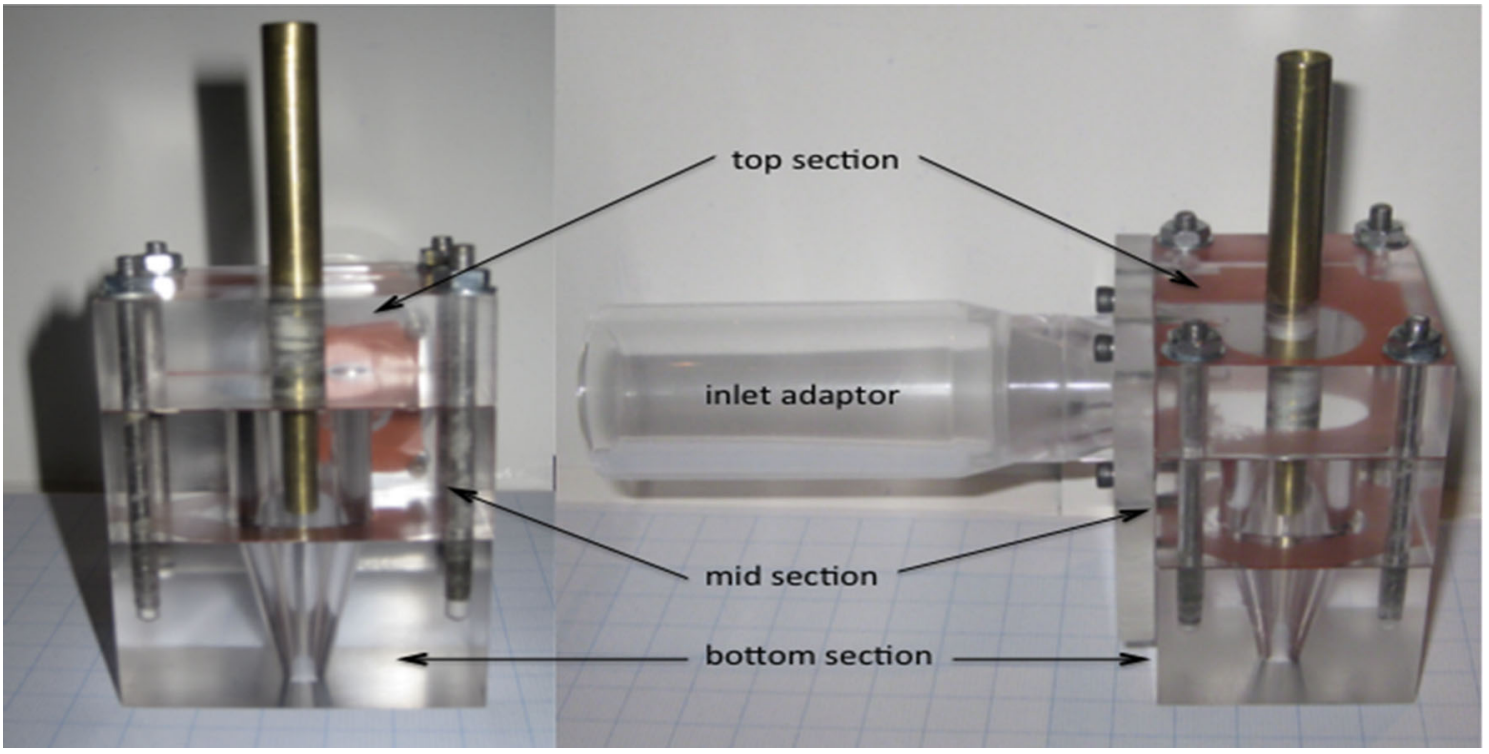

Fig. 3 Photograph of reverse flow cyclone spacer prototype (Cheng 2).

alone, $10 \mathrm{ml}$ of mobile phase were added at the first 5 stages, whilst $5 \mathrm{ml}$ of solvent was used for the last 3 stages of the impactor. All the stages were set on a laboratory rocker (Stirling Mixer, Sandrest Ltd, UK) and rocked for $2 \mathrm{~min}$ in order for the solvent to clean the entire surface. When the Cyclohaler ${ }^{\circledR}$ was employed with the Cheng 1, $5 \mathrm{ml}$ of mobile phase was added to all the stages. The concentration of the drug from each stage was determined by HPLC. After performing the experiments, each stage and the NGI were thoroughly cleaned with Millipore water and the coating was removed with acetone and rinsed with methanol.

\section{Aerosolization Assessment Using Next Generation Impactor for Cheng 2}

The second cyclone (Cheng 2) provided a lower resistance to airflow $\left(0.04 \mathrm{kPa}^{1 / 2}\right.$ min $\left.\mathrm{L}^{-1}\right)$ than Cheng $1(0.06$ $\mathrm{kPa}^{1 / 2}$ min $\left.\mathrm{L}^{-1}\right)$. Cyclohaler ${ }^{\circledR}$, Seretide (500)/Accuhaler ${ }^{\mathrm{TM}}$ and Easyhaler (400)/budesonide ${ }^{\mathrm{TM}}$ were employed with and without Cheng 2 at 2 and $4 \mathrm{kPa}$ to represent values achievable by patients. Each device with the Cheng 2 was connected to a dose uniformity sampling apparatus (DUSA, Copley Scientific Ltd., Nottingham, UK). The pressure drop was set accordingly and the respective flow rate was measured with a flow meter. $2 \mathrm{kPa}$ corresponded to $37 \mathrm{Lmin}^{-1}, 34 \mathrm{Lmin}^{-1}$ and $31 \mathrm{Lmin}^{-1}$ for the Cyclohaler ${ }^{\circledR}$, Seretide/Accuhaler ${ }^{\mathrm{TM}}$ and Easyhaler/ budesonide ${ }^{\mathrm{TM}}$ in series with the Cheng 2, respectively, and $4 \mathrm{kPa}$ to $51 \mathrm{Lmin}^{-1}, 50 \mathrm{Lmin}^{-1}$, and $43 \mathrm{Lmin}^{-1}$ for the Cyclohaler ${ }^{\circledR}$, Seretide/Accuhaler ${ }^{\mathrm{TM}}$ and Easyhaler/ budesonide $^{\mathrm{TM}}$, in series with the Cheng 2, respectively. Each DPI was also tested alone at the corresponding flow rates. Prior to performing the measurements with the NGI, the desired flow rate was adjusted with a flow meter. The NGI analysis and sample recovery were performed as above for Cheng 1 , with the exception of the respective mobile phase being employed in preseparator and for washing of the NGI stages.

\section{Aerosolization Studies Using Laser Diffraction Analysis}

Aerosolization of the active pharmaceutical ingredients (API) from each device was performed by laser diffraction analysis using the Sympatec Inhaler module (Inhaler Helos/KF, Sympatec Limited, Bury, UK) with flow rates between 30 and $60 \mathrm{Lmin}^{-1}$ from the Cyclohaler ${ }^{\circledR}$ device in absence and presence of Cheng 1 ; and at 2 and $4 \mathrm{kPa}$ for all the DPIs with and without Cheng 2.

\section{Data Analysis}

The $\%$ recovery was calculated, for full mass balance, as the total recovered drug $(\mu \mathrm{g})$ on each stage and the device as a $\%$ of the total known mass of the drug actuated (i.e. the dose content). The recovered dose (RD) was calculated as the sum of the dose $(\mu \mathrm{g})$ in each stage of the NGI and device or the NGI, device and spacer. The emitted dose (ED) was calculated as the sum of the total dose recovered from the NGI (or NGI and spacer) following actuation of the device. The spacer-emitted dose (sED) was calculated as the sum of the total dose recovered from the NGI only, following when the spacer was used. The $\%$ retention of the cyclone was calculated as the mass of drug deposited in the spacer as a percentage of the ED. The \% induction port/pre-separator (IP/PS) deposition was calculated as the mass of drug recovered on the IP/PS stage as a percentage of the ED or the sED. The $\%$ fine particle fraction below $5 \mu \mathrm{m}\left(\mathrm{FPF}_{5 \mu \mathrm{m}}\right)$ was calculated following interpolation of the cumulative 
particle size distribution of the dose deposited on the NGI stages as a percentage of the ED (i.e. ex device) or the sED (i.e. ex cyclone). The mass median aerodynamic diameter values (MMAD) were determined by interpolation of the cumulative particle size distribution of the dose deposited on the impactor stages.

\section{Statistical Analysis}

Statistical analysis was performed in Minitab using one-way ANOVA and post-hoc Tukey's test (multiple comparisons) or Student's two-tailed $t$-test for pair-wise comparisons, both at $95 \%$ confidence intervals.

\section{RESULTS}

\section{Aerosolization Studies with Next Generation Impactor Using Cheng I}

The emission of SS from Ventolin/Accuhaler ${ }^{\mathrm{TM}}$ formulation using a Cyclohaler ${ }^{\circledR}$ DPI was assessed between 30 and $60 \mathrm{Lmin}^{-1}$ with and without the Cheng 1 cyclone. The \% drug recovery was adequate at all the three flow rates with and without the cyclone ranging from $79.44 \pm 2.90 \%$ to $81.45 \pm$ $3.14 \%$ (Table I). The retention within the Cyclohaler $\AA$ did not change upon increasing the flow rate between 30 and $60 \mathrm{Lmin}^{-1}(25.98 \pm 8.07 \%$ and $25.95 \pm 5.95 \%$ of RD respectively, $p$ value $>0.05)$. When aerosolization was performed with the add-on spacer, altering the flow rate did not significantly alter the $\%$ deposited on the Cyclohaler ${ }^{\circledR}$ $\left(18.02 \pm 4.50 \%\right.$ to $21.51 \pm 3.70 \%$ at 30 and $60 \mathrm{Lmin}^{-1}$, respectively $p$ value $>0.05$, ANOVA). Deposition within Cheng 1 was high, although it did decrease with increasing flow rate (from $81.68 \pm 6.88 \%$ to $55.90 \pm 10.64 \%$, at 30 and $60 \mathrm{Lmin}^{-1}, p$ value $\left.<0.05\right)$
The results indicate that the presence of the cyclone increased the respirable fraction of the total dose which would be inhaled by patients (i.e. of the spacer-emitted dose, sED), also called fine particle fraction below $5 \mu \mathrm{m}\left(\mathrm{FPF}_{5 \mu \mathrm{m}}\right)$ (Table I). When the cyclone was employed a dramatically decreased induction port/pre-separator (IP/PS) deposition was seen $(p<0.05$, Table I). The extent of IP/PS deposition was unaffected by a change in the flow rate $(p>0.05)$ due (in part) to the variability between replicate analyses. Considering the aerodynamic particle size distribution (PSD) of the aerosol emitted into the impactor (Fig. 4), it was observed that the majority of the dose from the DPI alone deposited in the induction port/pre-separator (IP/PS)-the stages which collect non-respirable particles. Conversely, when the cyclone was employed the majority of the dose emitted from the spacer (i.e. the dose which would be inhaled by a patient) penetrated beyond the IP/PS to the sizeable stages. Figure 4 shows an unconventional representation of the cumulative undersize distribution. The calculation was based following the method of Thiel (30) and Mitchell et al. (31, 32). Typically the aerosol PSD is normalized for the impactor sizeable fraction only. However, such a conventional representation does not demonstrate the extent of the IP/PS deposition, which is visible in Fig. 4 in the distributions calculated as \% ED (blue and black plots).

The fine particle fractions $\left(\mathrm{FPF}_{5 \mu \mathrm{m}} \% \mathrm{ED}\right)$ of SS with $d_{a e}<$ $5 \mu \mathrm{m}$ are reported in Table I. The $\mathrm{FPF}_{5 \mu \mathrm{m}}$ from the Cyclohaler ${ }^{\circledR}$ increased from $20.83 \pm 3.00 \%$ to $33.84 \pm$ $2.90 \%$ when the flow rates were increased (ANOVA, $p$ value $<0.05)$. When the spacer was employed, considering the $\mathrm{FPF}_{5 \mu \mathrm{m}}(\% \mathrm{ED})$, i.e. as a $\%$ of the total dose emitted from the Cyclohaler ${ }^{\circledR}$ (Table I) there was no significant difference between 45 and $60 \mathrm{Lmin}^{-1}$. When the $\mathrm{FPF}_{5 \mu \mathrm{m}}$ was expressed as a $\%$ of the dose emitted from Cheng 1 (i.e. FPF $\%$ SED) it was clear that the majority of the inhalable aerosol was respirable, unlike when the Cyclohaler ${ }^{\circledR}$ was tested alone

Table I Aerosolisation of Salbutamol Sulphate from a Carrier Based Blend at 30, 45 and $60 \mathrm{Lmin}^{-1}$ (Mean \pm SD, $n \geq 4$ ) (IP/PS-Induction Port/Pre-Separator Deposition, MMAD-Mass Median Aerodynamic Diameter, FPF $5 \mu \mathrm{m}$ (ED)-Fine Particle Fraction $<5 \mu \mathrm{m}$ of the Emitted Dose)

\begin{tabular}{|c|c|c|c|c|c|c|c|}
\hline Device & $\begin{array}{l}\text { Flow rate } \\
\left(\operatorname{Lmin}^{-1}\right)\end{array}$ & $\begin{array}{l}\% \text { drug } \\
\text { recovery }\end{array}$ & $\begin{array}{l}\text { MMAD } \\
(\mu \mathrm{m})\end{array}$ & \multicolumn{2}{|c|}{ IP/PS deposition } & \multicolumn{2}{|c|}{$\% \mathrm{FPF}_{5 \mu \mathrm{m}}(\mathrm{ED})$} \\
\hline Cyclohaler® & 30 & $86.63 \pm 6.00$ & $3.07 \pm 0.26$ & \multicolumn{2}{|c|}{$70.22 \pm 3.23$} & \multicolumn{2}{|c|}{$20.83 \pm 3.00$} \\
\hline Cyclohaler® & 45 & $83.06 \pm 3.59$ & $2.49 \pm 0.22$ & \multicolumn{2}{|c|}{$66.34 \pm 3.38$} & \multicolumn{2}{|c|}{$27.86 \pm 3.50$} \\
\hline \multirow[t]{2}{*}{ Cyclohaler ${ }^{\circledR}$} & \multirow[t]{2}{*}{60} & \multirow[t]{2}{*}{$81.50 \pm 4.01$} & \multirow[t]{2}{*}{$1.98 \pm 0.09$} & \multicolumn{2}{|c|}{$61.74 \pm 3.02$} & \multicolumn{2}{|c|}{$33.84 \pm 2.90$} \\
\hline & & & & $\begin{array}{l}\text { Expressed as \% of } \\
\text { dose emitted DPI } \\
(\% E D)\end{array}$ & $\begin{array}{l}\text { Expressed as \% of } \\
\text { dose emitted from } \\
\text { Cheng I (\%sED) }\end{array}$ & $\begin{array}{l}\text { Expressed as \% of } \\
\text { dose emitted DPI } \\
(\% E D)\end{array}$ & $\begin{array}{l}\text { Expressed as \% of } \\
\text { dose emitted from } \\
\text { Cheng I (\%sED) }\end{array}$ \\
\hline With Cheng I & 30 & $79.44 \pm 2.90$ & $0.90 \pm 0.06$ & $2.45 \pm 1.18$ & $14.82 \pm 7.80$ & $\mid 4.48 \pm 5.84$ & $82.75 \pm 7.34$ \\
\hline With Cheng I & 45 & $78.52 \pm 2.27$ & $0.76 \pm 0.04$ & $2.32 \pm 2.77$ & $6.95 \pm 7.91$ & $26.44 \pm 4.34$ & $92.24 \pm 7.74$ \\
\hline With Cheng I & 60 & $78.43 \pm 4.31$ & $0.76 \pm 0.14$ & $9.56 \pm 6.05$ & $22.27 \pm 9.96$ & $33.42 \pm 5.86$ & $77.00 \pm 10.07$ \\
\hline
\end{tabular}




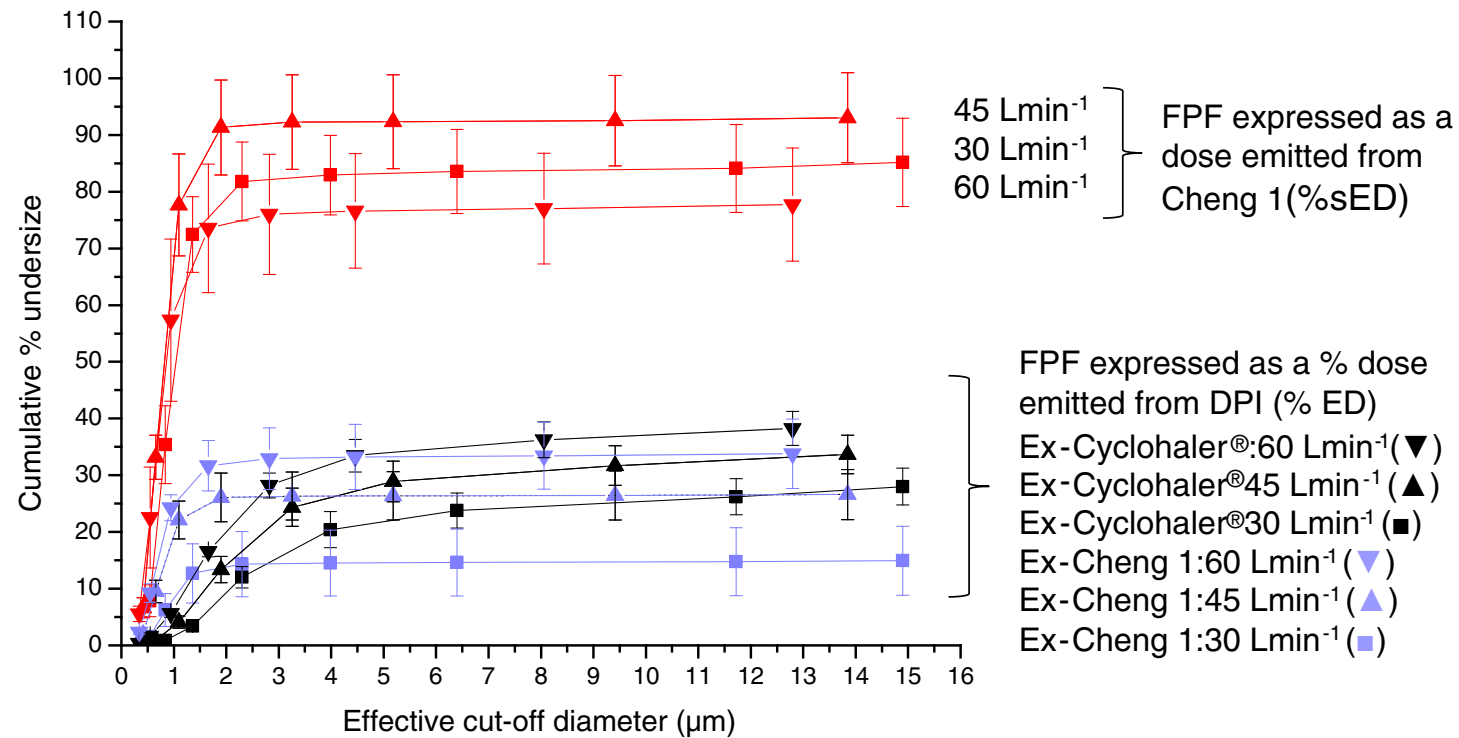

Fig. 4 Cumulative aerodynamic undersize (\%) of salbutamol sulphate of total emitted dose from Cyclohaler $囚$ at $30 \mathrm{Lmin}^{-1}(\mathbf{\bullet}), 45 \mathrm{Lmin}^{-1}$ ( $\left.\mathbf{\Delta}\right)$ and $60 \mathrm{Lmin}^{-1}$

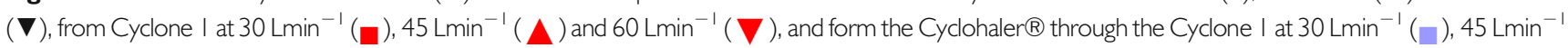

$(\triangle)$ and $60 \mathrm{Lmin}^{-1}(\nabla)$. (mean $\left.\pm \mathrm{SD} ; n \geq 4\right)$.

(Table I, $p<0.05)$. The $\mathrm{FPF}_{5 \mu \mathrm{m}}(\% \mathrm{osED})$ was not significantly affected by flow rate $(p>0.05)$. However, a high variability was seen in the $\mathrm{FPF}_{5 \mu \mathrm{m}}$ at the highest flow rate $(77.00 \pm 10.07 \%)$. The median aerodynamic diameter (MMAD) values (Table I) were $\sim 3$ times smaller with Cheng 1 in place than for the Cyclohaler® alone (ANOVA, post-hoc Tukey's test, $p<0.05)$. The MMADs with the cyclone in place did not change upon altering the flow rate (ANOVA, $p>0.05$ ). Thus the cyclone served to reduce potential oropharyngeal deposition (i.e. IP/ PS deposition) whilst limiting particle output to the respirable range, owing to the aerodynamic fractionation of the cyclone.

\section{Particle Size Distribution for Cheng I Using Laser Diffraction Analysis}

In order to understand the lower $\mathrm{FPF}_{5 \mu \mathrm{m}}$ and the variability in IP/PS deposition with the cyclone at $60 \mathrm{Lmin}^{-1}$, laser diffraction analysis (LDA) on the emitted aerosol cloud was performed using the Sympatec Inhaler module. LDA also displayed the potential of the cyclone to retain large agglomerates of micronized drug and carrier particles. The volume median diameter $\left(\mathrm{Dv}_{50}\right)$ of the aerosol cloud released from the cyclone at $30 \mathrm{Lmin}^{-1}$ was $1.03 \pm 0.01 \mu \mathrm{m}$ which was of the same order of magnitude as the MMAD and substantially lower than when the cyclone was absent $(40.04 \pm$ $4.03 \mu \mathrm{m})$. The same trend was seen at 45 and $60 \mathrm{Lmin}^{-1}$ when the $\mathrm{DV}_{50}$ was $1.18 \pm 0.03 \mu \mathrm{m}$ and $0.96 \mu \mathrm{m}$ respectively. At the higher flow rate, variability was observed in the particle size distributions of the aerosol emitted from the cyclone (an example of two size distribution measurements at $60 \mathrm{Lmin}^{-1}$ are presented in Fig. 5). The bi-modal PSD indicated that some agglomerates and/or carrier particles were observed to escape the cyclone at the highest flow rate which would have resulted in IP/PS deposition (only $\sim 50 \%$ of the volume distribution was $<3 \mu \mathrm{m}$ ). However, the latter observations of agglomerate emission displayed variability from actuation to actuation.

\section{Aerosolization Studies Using Cheng 2-Compatibility with Marketed Products}

The Cheng 1 cyclone offered excessive resistance to inhalation

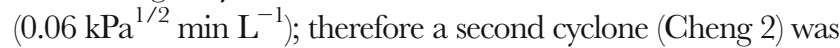
designed with a lower resistance $\left(0.04 \mathrm{kPa}^{1 / 2} \mathrm{~min} \mathrm{~L}^{-1}\right)$. The Aerosolization of SS from the Cyclohaler ${ }^{\circledR}$, Bud from the Easyhaler/Budesonide $^{\mathrm{TM}}$ and SX/FP from the Seretide/ Accuhaler ${ }^{\mathrm{TM}}$ was assessed with and without Cheng 2. In order to best simulate the way in which the combined spacer and DPI would be used by a patient, testing was performed at the flow rates necessary to achieve a pressure drop of either 2 or $4 \mathrm{kPa}$ across the two in series to study dose emission. $2 \mathrm{kPa}$ corresponded to a flow of $37 \mathrm{Lmin}^{-1}, 34 \mathrm{Lmin}^{-1}$ and $31 \mathrm{Lmin}^{-1}$ through the Cyclohaler ${ }^{\circledR}$, Accuhaler ${ }^{\mathrm{TM}}$ and Easyhaler ${ }^{\mathrm{TM}}$, respectively. $4 \mathrm{kPa}$ corresponded to flows of $51 \mathrm{Lmin}^{-1}$, $50 \mathrm{Lmin}^{-1}$, and $43 \mathrm{Lmin}^{-1}$ through the Cyclohaler ${ }^{\circledR}$, Accuhaler ${ }^{\mathrm{TM}}$ and Easyhaler ${ }^{\mathrm{TM}}$, respectively. The percentage recoveries for all the APIs with and without the Cheng 2 were within pharmacopoeial limits (Table II). The Cheng 2 showed high retention of non-respirable drug as observed for Cheng 1 (Table II) although no change was detected when the flow rate increased $(p>0.05)$.

The high retention in the spacer decreased the deposition of the particles in the induction port/pre-separator stages (Table II) 


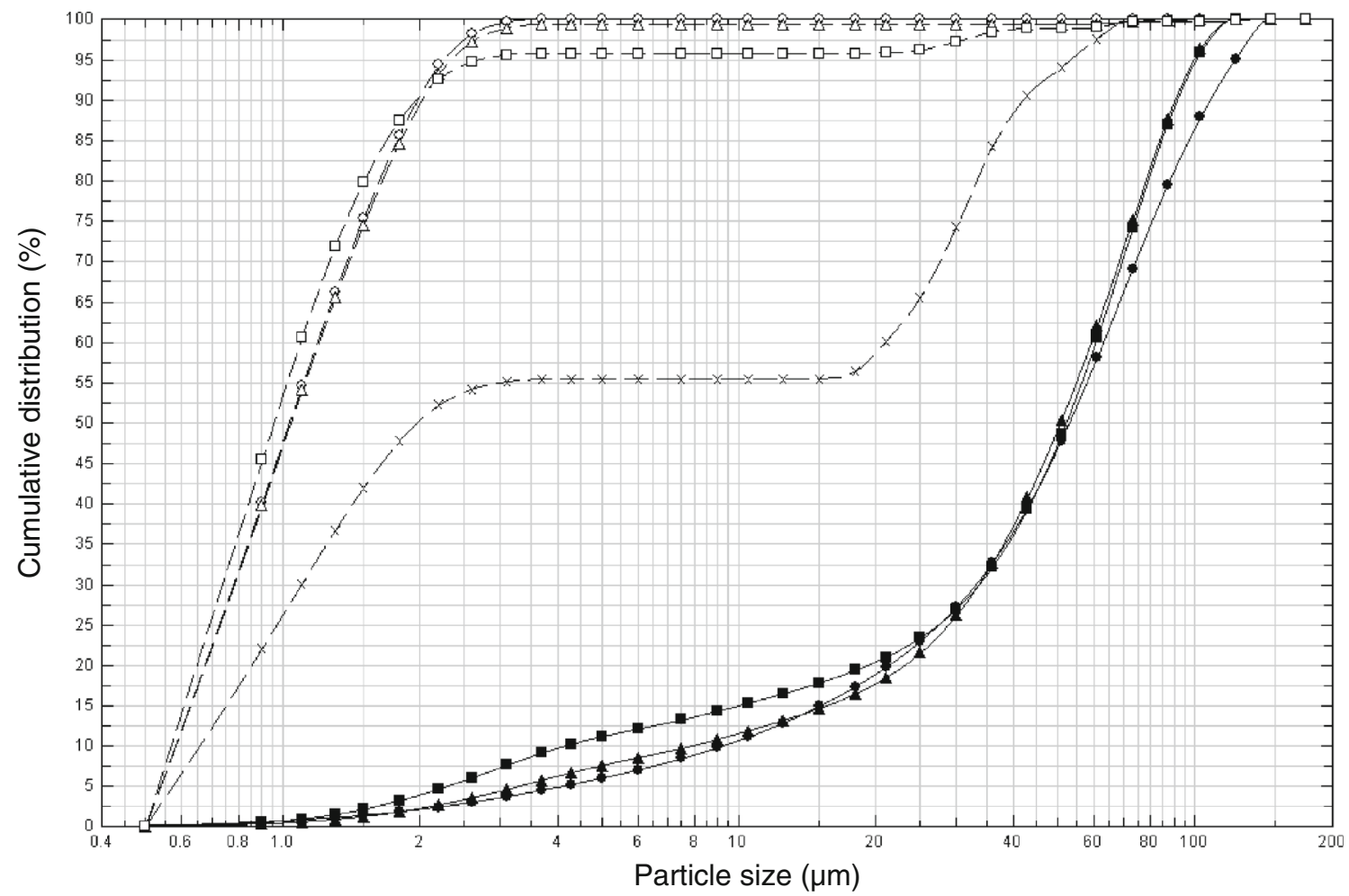

Fig. 5 Representative particle size distributions of salbutamol sulphate from the Cyclohaler ${ }^{\circledR}$ alone at $30 \mathrm{Lmin}^{-1}$ (black circle), $45 \mathrm{Lmin}{ }^{-1}$ (black up-pointing triangle) and $60 \mathrm{Lmin}^{-1}$ (black square) and with the Cheng I at $30 \mathrm{Lmin}^{-1}$ (white circle), $45 \mathrm{Lmin}^{-1}$ (white up-pointing triangle) and $60 \mathrm{Lmin}^{-1}$ (white square). The data show the absence of the coarse lactose fraction when the spacer was used and the appearance of some agglomerates emitted from the Cheng I ( $x$ ) at $60 \mathrm{Lmin}^{-1}$ causing a bi-modal distribution.

compared to when the devices were tested on their own. In the case of Cyclohaler ${ }^{\circledR} / \mathrm{SS}$ and Easyhaler/Budesonide ${ }^{\mathrm{TM}}$, increasing the flow rate did not alter the IP/PS deposition $(p>$ $0.05)$ both with and without the cyclone in place. The retention of Bud within the Cheng 2 cyclone was lower than that of SS
(Table II, $p<0.05)$. When the SX and FP were tested from the Seretide/AccuhalerTM, the IP/PS deposition was higher than that of the SS and Bud at both flow rates $(p<0.05$, Table II). IP/PS deposition of SX/FP remained higher than SS and Bud when the cyclone was used. In addition to the reduction of the

Table II Values of \% Drug Recovery, \% Induction Port/Pre-Separator (IP/PS) Deposition and \% Retention Within the Cheng 2 Cyclone for Salbutamol Sulphate (SS), Budesonide (Bud), Salmeterol Xinafoate (SX) and Fluticasone Propionate (FP) Emitted from the Cyclohaler ${ }^{\circledR}$, Easyhaler ${ }^{\text {TM }}$ and Accuhaler ${ }^{T M}$, Respectively at 2 and $4 \mathrm{kPa}$ (Mean $\pm \mathrm{SD}>4$ ), ED is the Dose Emitted from the DPI and sED is the Dose Emitted from the Spacer

\begin{tabular}{|c|c|c|c|c|c|c|c|c|}
\hline \multirow[t]{2}{*}{ Drug } & \multirow{2}{*}{$\begin{array}{l}\text { Device } \\
\text { Pressure drop }\end{array}$} & \multicolumn{2}{|l|}{$\%$ drug recovery } & \multicolumn{2}{|c|}{ Retention in the cyclone(\% ED) } & & \multicolumn{2}{|c|}{ IP/PS deposition } \\
\hline & & $2 \mathrm{kPa}$ & $4 \mathrm{kPa}$ & $2 \mathrm{kPa}$ & $4 \mathrm{kPa}$ & & $2 \mathrm{kPa}$ & $4 \mathrm{kPa}$ \\
\hline \multirow[t]{3}{*}{ SS } & Cyclohaler® & $85.68 \pm 1.20$ & $82.14 \pm 2.19$ & & & As \% ED & $67.28 \pm 2.09$ & $64.44 \pm 2.49$ \\
\hline & Cyclone-Cyclohaler ${ }^{\circledR}$ & $82.29 \pm 3.98$ & $79.66 \pm 4.43$ & $87.61 \pm 2.96$ & $85.69 \pm 1.11$ & As \% ED & $2.22 \pm 1.51$ & $2.66 \pm 0.59$ \\
\hline & & & & & & As \% sED & $15.87 \pm 6.76$ & $18.44 \pm 2.79$ \\
\hline \multirow[t]{3}{*}{ Bud } & Easyhaler $^{\top \mathrm{M}}$ & $97.07 \pm 3.78$ & $105.48 \pm 3.69$ & & & As \% ED & $62.01 \pm 2.52$ & $63.18 \pm 3.72$ \\
\hline & Cyclone-Easyhaler ${ }^{\mathrm{TM}}$ & $|04.6| \pm 5.70$ & $104.50 \pm 4.66$ & $75.23 \pm 1.70$ & $75.12 \pm 2.94$ & As \% ED & $3.89 \pm 0.61$ & $3.73 \pm 0.59$ \\
\hline & & & & & & As \% sED & $15.65 \pm 1.39$ & $15.19 \pm 1.74$ \\
\hline \multirow[t]{3}{*}{ SX } & Accuhaler ${ }^{\top M}$ & $88.94 \pm 9.10$ & $97.73 \pm 2.96$ & & & As \% ED & $78.27 \pm 0.54$ & $76.93 \pm 2.39$ \\
\hline & Cyclone-Accuhaler ${ }^{\top \mathrm{M}}$ & $89.56 \pm 10.58$ & $101.25 \pm 13.56$ & $80.48 \pm 0.56$ & $83.05 \pm 3.67$ & As \% ED & $7.53 \pm 0.93$ & $6.13 \pm 2.68$ \\
\hline & & & & & & As \% sED & $38.70 \pm 4.56$ & $36.01 \pm 9.77$ \\
\hline \multirow[t]{3}{*}{ FP } & Accuhaler ${ }^{\top M}$ & $90.99 \pm 7.92$ & $|15.6| \pm 7.16$ & & & As \% ED & $78.21 \pm 1.87$ & $75.53 \pm 3.50$ \\
\hline & Cyclone-Accuhaler ${ }^{\mathrm{TM}}$ & $82.39 \pm 4.43$ & $102.68 \pm 12.07$ & $82.01 \pm 1.18$ & $85.70 \pm 2.28$ & As \% ED & $6.38 \pm 0.46$ & $4.15 \pm 1.19$ \\
\hline & & & & & & As \% sED & $35.03 \pm 1.45$ & $29.63 \pm 5.85$ \\
\hline
\end{tabular}


IP/PS deposition with Cheng 2, the $\mathrm{FPF}_{5 \mu \mathrm{m}}$ of the dose which would be inhaled by the patient significantly improved (Fig. 6b, compare $\mathrm{FPF}_{5 \mu \mathrm{m}} \% \mathrm{ED}$ and $\left.\mathrm{FPF}_{5 \mu \mathrm{m}} \% \mathrm{sED},(p<0.05)\right)$. All the inhalers exhibited flow-rate dependence of the $\% \mathrm{FPF}_{5 \mu \mathrm{m}}$ when employed alone (Fig. 6b). Easyhaler/Budesonide ${ }^{\mathrm{TM}}$ was the only product where the change in flow rate altered the $\mathrm{FPF}_{5 \mu \mathrm{m}}(p<$ $0.05)$ when the cyclone was employed.

The $\mathrm{FPF}_{5 \mu \mathrm{m}} \% \mathrm{osED}$ differed significantly between the products at both flow rates $\left(\mathrm{SX}\right.$ Accuhaler ${ }^{\mathrm{TM}}<\mathrm{FP}$ Accuhaler $^{\mathrm{TM}}<$ Bud Easyhaler ${ }^{\mathrm{TM}}=$ SS Cyclohaler $\left.{ }^{\circledR}, p<0.05\right)$. This was similar to the behaviour of the devices when tested without the cyclone (i.e. comparing $\mathrm{FPF}_{5 \mu \mathrm{m}} \% \mathrm{ED}$ ). When the cyclone was employed the fine particle dose $(<5 \mu \mathrm{m}$, FPD) was unaffected by a change in flow rate $(p>0.05)$, with the exception of the Easyhaler/Budesonide ${ }^{\mathrm{TM}}(p<0.05)$. However, with the exception of SX, the FPDs of all inhaled drugs were significantly lower $(p<0.05)$ when Cheng 2 was employed (Fig. 6c). By way of example, SS combined with Cheng $2(15.13 \pm 4.78 \mu \mathrm{g}$ and 13.77 $\pm 1.67 \mu \mathrm{g}$ at 2 and $4 \mathrm{kPa}$, respectively) was lower than from the Cyclohaler ${ }^{\circledR}$ alone $(31.64 \pm 2.70 \mu \mathrm{g}$ and $36.14 \pm 5.04 \mu \mathrm{g}$ at 2 and $4 \mathrm{kPa}$, respectively).
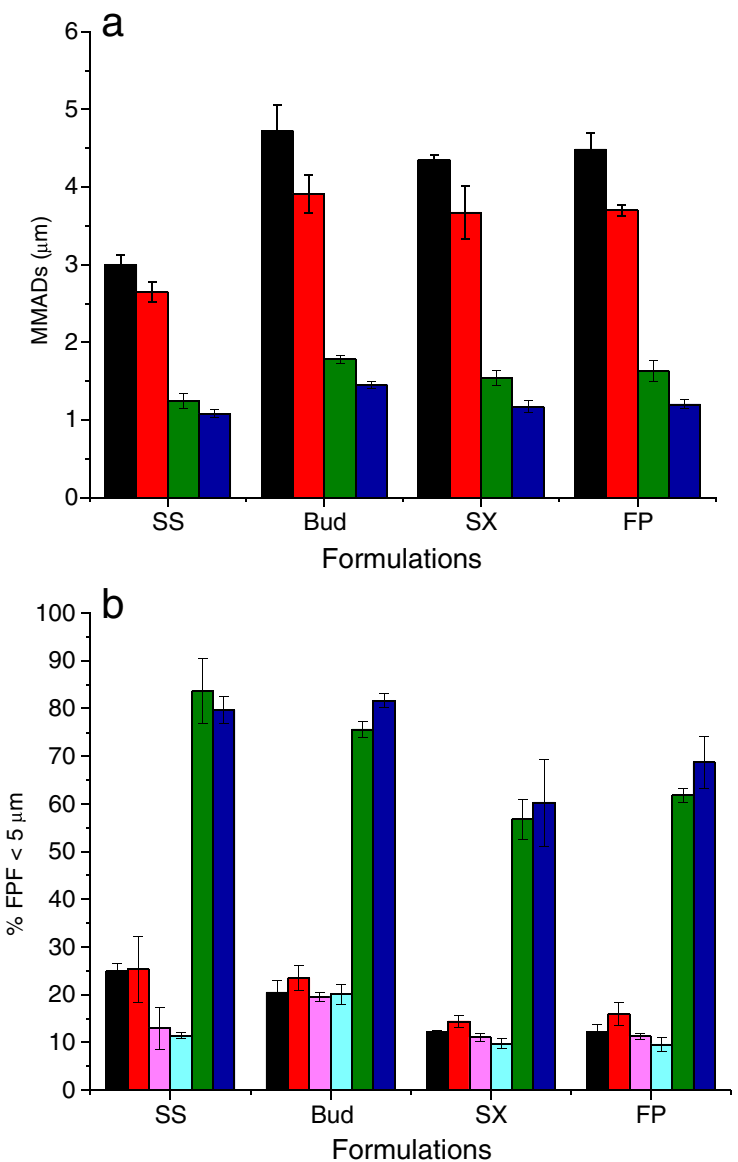

When the DPIs were tested alone, the MMADs decreased markedly when the flow rate was increased (Fig. 6a, $p<0.05$ ). The aerosols which would be inhaled by the patient (sED) possessed lower MMADs when emitted from the cyclone than from the devices alone (Fig. 6a, $p<0.05$ ). For example, the MMAD of SS was halved when the cyclone was employed (Cyclohaler®: 3.00 $\pm 0.12 \mu \mathrm{m}$ and Cheng 2: $1.24 \pm 0.10 \mu \mathrm{m}$ at $2 \mathrm{kPa}$ ). This showed the screening ability of Cheng 2, however unlike Cheng 1, the MMADs were lower for all formulations at the $4 \mathrm{kPa}$ than $2 \mathrm{kPa}$ flow rate with the cyclone in place (Fig. 6a, $p<0.05$ ). The latter differences were not as large in magnitude as when the DPIs were tested without the spacer.

\section{Particle Size Distribution for Cheng 2 Using Laser Diffraction Analysis}

LDA was performed on the aerosol emitted from the Cyclohaler ${ }^{\circledR}$, Seretide/Accuhaler ${ }^{\mathrm{TM}}$ and Easyhaler/ Budesonide $^{\mathrm{TM}}$ with and without Cheng 2 at 2 and $4 \mathrm{kPa}$. The $\mathrm{Dv}_{50}$ was dramatically decreased when Cheng 2 was employed for all the three different DPIs in the same manner of Cheng 1
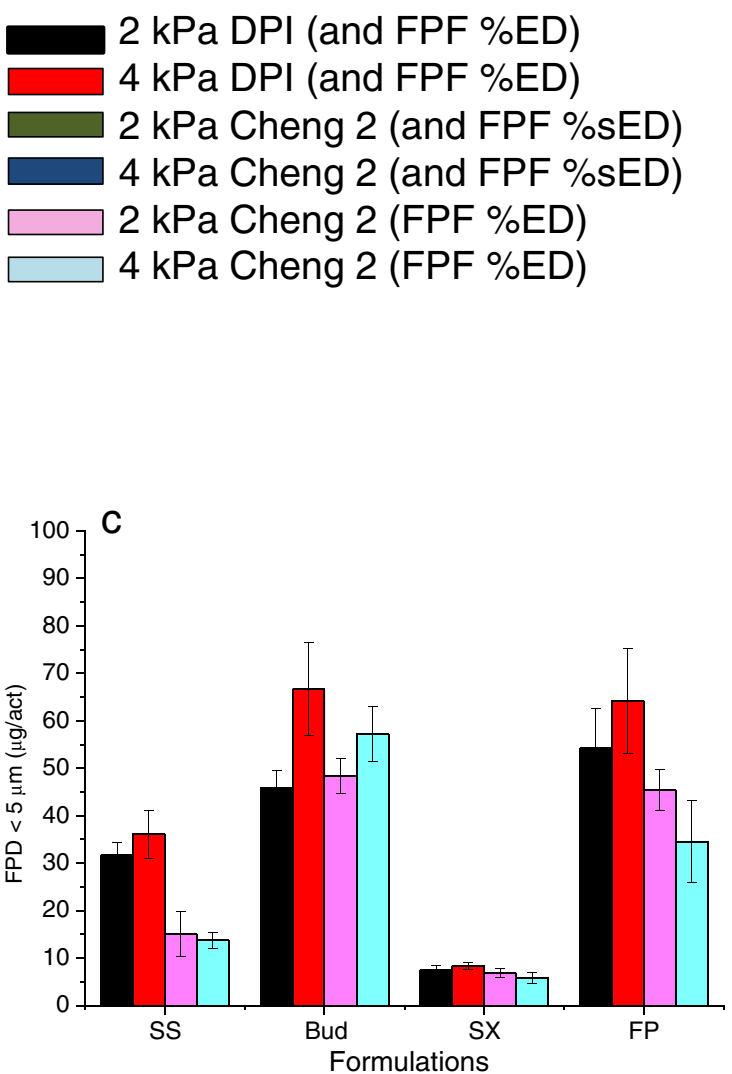

Fig. 6 (a) Mass median aerodynamic diameter (MMAD), (b) fine particle fraction $<5 \mu \mathrm{m}$ (FPF $5 \mu \mathrm{m}$ ), and (c) fine particle dose $<5 \mu \mathrm{m}\left(\mathrm{FPD}_{5 \mu \mathrm{m}}\right)$; of salbutamol sulphate (SS), budesonide (Bud), salmeterol xinafoate (SX) and fluticasone propionate (FP) emitted from the DPIs and from the DPls through the Cheng 2 cyclone, (mean $\pm S X, n \geq 4$ ). 
(Table III). With Seretide/Accuhaler ${ }^{\mathrm{TM}}$ escape of agglomerates from the cyclone was observed, although the $\mathrm{Dv}_{50}$ values were much reduced $(1.73 \pm 0.16$ and $1.15 \pm 0.12 \mu \mathrm{m}$, at 2 and $4 \mathrm{kPa}$, respectively) compared to the Seretide/Accuhaler ${ }^{\mathrm{TM}}$ alone. Similar to the variability in agglomerate emission from actuation to actuation with Cheng 1 at high flow rates, a shift in PSD to larger sizes was observed with Seretide/Accuhaler ${ }^{\text {TM }}$ (indicated by the arrows in Fig. 7).

\section{DISCUSSION}

The aim of this study was to examine whether reverse-flow cyclone devices are a suitable approach as a generic spacer to overcome some of the practical and clinical difficulties of DPI use. The latter difficulties include: excessive oropharyngeal deposition (8) leading to side-effects such as dysphonia and candidiasis (10); dependence of the respirable dose on the inhalation flow rate $(4,33,34)$; dependence of the aerodynamic diameter on the inhalation flow rate (15). A DPI containing a miniature reverse cyclone (Conix ${ }^{\text {TM}}$ ) has shown promise of increased FPF compared to a comparator device at $4 \mathrm{kPa}(26)$. We have attempted to extend the possible benefits of improved FPFs by designing a generic platform spacer which could be used by patients to reduce the oropharyngeal deposition regardless of their prescribed DPI.

The two cyclone configurations designed from CFD studies $(27,28)$ that sought to minimize device resistance and achieve an emission limited to the finest, respirable particles. The investigations centred around three carrier-based formulations: SS as Ventolin ${ }^{\mathrm{TM}}$ aerosolized using the Cyclohaler ${ }^{\circledR}$ device; Bud aerosolized from the Easyhaler/ Budesonide $^{\mathrm{TM}}$; and SX and FP aerosolized using the Seretide/Accuhaler ${ }^{\mathrm{TM}}$. The latter list covers a variety of particle physicochemical properties aerosolized from devices covering the single-dose capsule, multiple single-dose and reservoir multiple-dose platforms.

Table III Values of Volume Median Diameter ( $\left.\mathrm{DV}_{50}\right)$ of the Aerosol Clouds Emitted from the Dry Powder Inhalers with and without Cheng 2 (Mean \pm $\mathrm{SD}, \mathrm{n}=3$ )

\begin{tabular}{llrr}
\hline API & Device & \multicolumn{2}{c}{$\mathrm{DV}_{50}(\mu \mathrm{m})$} \\
\cline { 3 - 4 } Pressure drop & & \multicolumn{1}{c}{$2 \mathrm{kPa}$} & \multicolumn{1}{c}{$4 \mathrm{kPa}$} \\
\hline SS & Cyclohaler $^{\circledR}$ & $58.66 \pm 3.42$ & $61.56 \pm 3.18$ \\
& Cyclone-Cyclohaler $^{\circledR}$ & $1.29 \pm 0.07$ & $1.13 \pm 0.02$ \\
Bud & Easyhaler $^{\text {TM }}$ & $32.68 \pm 2.09$ & $31.14 \pm 2.12$ \\
& Cyclone-Easyhaler $^{\text {TM }}$ & $1.88 \pm 0.02$ & $1.68 \pm 0.05$ \\
SX/FP & Accuhaler $^{\text {TM }}$ & $48.13 \pm 2.03$ & $46.01 \pm 2.99$ \\
& Cyclone-Accuhaler $^{\text {TM }}$ & $1.73 \pm 0.13$ & $1.15 \pm 0.12$ \\
& & &
\end{tabular}

The data were obtained by laser diffraction analysis and identify the absence the coarse carrier in the aerosol emitted from the cyclone
Both spacers were successful in terms of reducing potential oropharyngeal deposition of the dose emitted from the device as shown in Tables I and II. It was interesting that when the cyclone was employed, the IP/PS deposition was not altered regardless of flow rate, and was always below half that of the DPI alone. Adequate mass balance was achieved throughout all experiments (i.e. \% recovered doses were within pharmacopoeial limits), and the explanation for the reduced IP/PS deposition was due to retention of the drug in the cyclone spacer (e.g. $81.68 \pm 6.88 \%$ of SS at $30 \mathrm{Lmin}^{-1}$ from Cheng 1). Retention of drug within the spacer is a common feature when spacers are employed with MDIs $(35,36)$ and is beneficial in that oropharyngeal deposition is consequently reduced. It was interesting to note that neither cyclone retention nor IP/PS deposition was affected by the flow rate for any of the drug-device formulations tested in the current study. The IP/PS deposition was unaltered for SS whether Cheng 1 or Cheng 2 was employed as the spacer. Although the extent of IP/PS deposition and cyclone retention did depend on the drug formulation/product type (Table II), the potential for flow-rate independence of oropharyngeal drug deposition for a specific formulation was a promising finding towards overcoming inter-patient variability in DPI deposition profiles.

The mass median aerodynamic diameter (MMAD) is one determinant (alongside inhalation parameters) of the potential deposition site of an inhaled aerosol. It would be expected that the MMADs for the aerosols emitted from the spacers would be limited by the effective cut-off diameters of the cyclone itself $(27,28)$ because the cyclone acts as a classifier, releasing only particles smaller than the cut-off size. The MMAD values (Table I) for SS with Cheng 1 in place were $\sim 3$ times smaller than for the Cyclohaler® itself (ANOVA, post-hoc Tukey's test, $p<0.05)$. Similar observations of reduction in the MMAD were seen with Cheng 2 for all drugs studied, albeit ranging between 2 and 3 fold reductions. Indeed the MMADs were roughly equivalent to the calculated cut-off diameters of the cyclones (Cheng 1 0.9 $\mu \mathrm{m}$, Cheng 2 2 $\mu \mathrm{m}$ ).

It appeared that the cyclone spacers did function effectively as classifiers, a finding confirmed by laser diffraction analysis (Figs. 5 and 7 and Table III). For both spacers, it was clear that the non-respirable lactose fraction was retained in the spacer, and the particle size output was limited (with some exceptions discussed below) to particles with a geometric diameter below the spacer cut-offs. Accepting that a change in MMAD compared to the device alone is not desirable and challenges equivalence of drug delivery, it is important to consider several points. First, it appears that the MMAD was limited by the effective cut-off diameter of the cyclone, and hence the possibility for design optimization. Second, spacer use with MDIs (although clinically accepted) is also associated with altered MMADs and lung deposition fractions $(37,38)$. Finally, it is accepted with DPIs that patients currently inhale aerosols with a range of MMADs arising from their different 


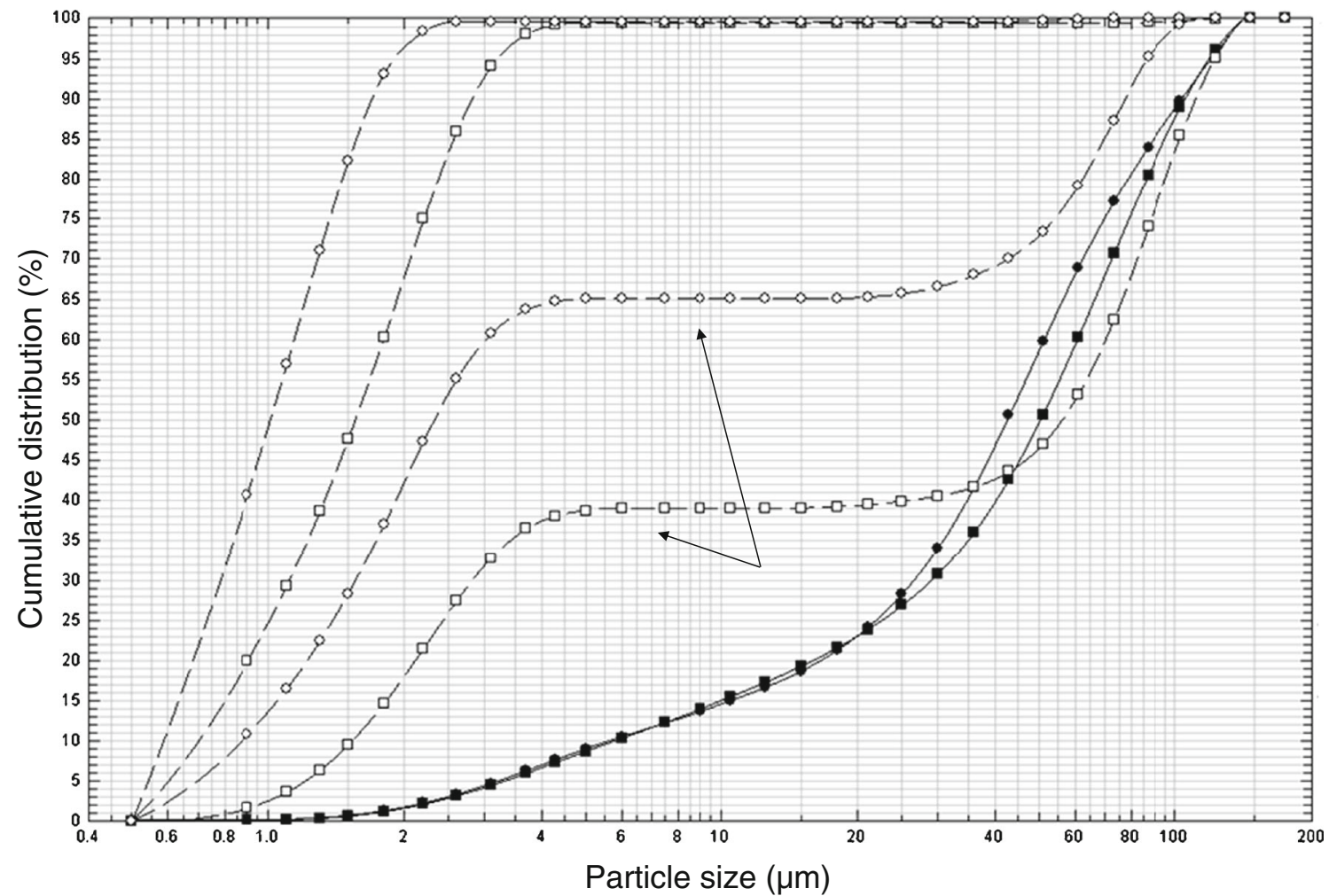

Fig. 7 Representative particle size distribution of salmeterol xinafoate- and fluticasone propionate-containing aerosol clouds from Seretide/Accuhaler ${ }^{\top \mathrm{M}}$ at $2 \mathrm{kPa}$ (black square) and $4 \mathrm{kPa}$ (black circle) and from Cheng 2 at $2 \mathrm{kPa}$ (white square) and $4 \mathrm{kPa}$ (white circle). The data show the absence of the coarse lactose fraction when the spacer was used and the emission of agglomerates from the cyclone (indicated by arrows) for salmeterol xinafoate and fluticasone propionate blends at $2 \mathrm{kPa}$ (white square) and $4 \mathrm{kPa}$ (white circle).

inhalation performance $(15,18)$. It appears that particularly with Cheng 1, the use of the cyclone minimized the changes in the MMAD upon varying the flow rate observed with SSCyclohaler® alone. This represents a possible advantage for the use of a spacer with the DPI.

DPI spacers have been previously shown to reduce potential oropharyngeal deposition $(22,23)$, however they have not gained market acceptance. This may arise from the enhanced deagglomeration shown by some workers $(22,23)$ and the specificity of the spacers to a particular device (Turbuhaler TM) (20-23). The traditional view of spacer use with MDIs is that extra-thoracic deposition is reduced but that the fine particle deposition is unchanged when a spacer is employed (39). The target with a DPI is therefore similar. It is necessary to compare the $\mathrm{FPF}$ as a $\% \mathrm{ED}$ (i.e. the dose exiting the device) and examine its equivalency when the cyclone is used. The FPF $(\% \mathrm{ED})$ of SS ranged from $20.83 \pm 3.00 \%\left(30 \mathrm{Lmin}^{-1}\right)$ to $33.84 \pm 2.90 \%\left(60 \mathrm{Lmin}^{-1}\right)$ from the Cyclohaler ${ }^{\circledR}$ (Table I). With the exception of the lowest flow rate $\left(30 \mathrm{Lmin}^{-1}\right)$, there was no difference between the FPF $(\% \mathrm{ED})$ for the Cyclohaler ${ }^{\circledR}$ and the Cyclohaler ${ }^{\circledR}$-Cheng 1 combination. As well as desiring consistency in the FPF (\%ED) when the spacer is employed, it is also important to consider the FPF (\%sED), because this indicates the dose which would be inhaled by the patient. The benefit of the using the spacer is clear from Fig. 4 which indicates that the majority of the aerosol emitted from Cheng 1 was respirable (ranging between $82.75 \pm 7.34 \%$ and $77.00 \pm 10.07 \%$ at 30 and $60 \mathrm{Lmin}^{-1}$ ). The higher value of FPF $(\% \mathrm{sED})$ compared to FPF $(\% \mathrm{ED})$ indicates the ability of the spacer to remove the non-inhalable fraction of the aerosol emitted from the Cyclohaler ${ }^{\circledR}$ at a particular flow rate. The similarity of the values for 45 and $60 \mathrm{Lmin}^{-1}$ were promising in that the FPF appeared to be less affected by the alteration of the flow rate with the cyclone in place (Table I) and this represents an improvement over findings with previous DPI spacers (21-23).

The promising findings with Cheng 1 were achieved using a spacer with a cut-off diameter of $\sim 0.9 \mu \mathrm{m}$, but a resistance to airflow of $\left(0.06 \mathrm{kPa}^{1 / 2} \operatorname{min~L^{-1})}\right.$ which is excessive. Following CFD studies, the second Cheng 2 spacer was prototyped with an inlet diameter which reduced resistance $\left(0.04 \mathrm{kPa}^{1 / 2}\right.$ $\left.\min \mathrm{L}^{-1}\right)$, with the compromise of a higher cut-off diameter $(\sim 2 \mu \mathrm{m})$, albeit still in the respirable range. This level of resistance was deemed to be appropriate because it is in the centre of the range of other high resistance devices, e.g. Turbuhaler TM $\left(0.03 \mathrm{kPa}^{1 / 2}\right.$ min $\left.\mathrm{L}^{-1}\right)$ and Handihaler ${ }^{\mathrm{TM}}$ $\left(0.05 \mathrm{kPa}^{1 / 2} \operatorname{min~} \mathrm{L}^{-1}\right)(14,40)$. The promising findings of the reduced IP/PS (and hence potential oropharyngeal deposition) were accompanied by a comparable FPF ( $\% \mathrm{ED})$ with and without the cyclone. Although the FPF of SS was 
approximately halved by the use of the cyclone $(p<0.05)$, with the exception of $\mathrm{SX}$ and $\mathrm{FP}$ at the highest flow rate $(4 \mathrm{kPa}$, slightly lower, $p<0.05)$, there were no significant differences in the FPF $(\% \mathrm{ED})$ for Bud, SX or FP when the cyclone was added to the DPI product (Fig. 6b). The latter findings were also reflected in the fine particle dose (FPD) values (Fig. 6c). As well as the comparability (excluding SS) to the DPI results, it was important to note that using the cyclone minimized (or removed) the magnitude of change in FPD and FPF (\%ED), caused by increasing the flow rates.

Differences in the FPF (\% ED) when the cyclone is used indicate pharmaceutical non-equivalence. However, it is also important to consider the FPF $(\% \mathrm{sED})$-i.e. the fraction of the aerosol that would be inhaled by the patient when of assessing the suitability of the cyclone device as a generic spacer. The cyclone was designed to emit a similar $\mathrm{FPF}_{5 \mu \mathrm{m}}(\% \mathrm{sED})$ from all products. There were clear differences in the FPF (\% sED) values observed in this study. The highest FPFs were seen for SS and Bud (almost 80\% of the dose emitted from the spacer). A lower $\mathrm{FPF}_{5 \mu \mathrm{m}}$ was observed for $\mathrm{SX}$ and FP from the Seretide/ Accuhaler $^{\mathrm{TM}}$ (range 60-70\%) which was due to the higher IP/PS deposition of SX and FP compared to Bud and SS. Nevertheless, the Cheng 2 cyclone delivered an aerosol which had a reduced potential for oropharyngeal deposition (i.e. higher FPF \%sED) compared to all three DPI products tested (FPF \%ED). Such a reduction in IP/PS deposition is in accordance with measurements for SS from the Conix ${ }^{\mathrm{TM}}$ DPI (26). The current study has extended this showing the feasibility to combine a generic spacer with several marketed DPI formulations or devices to achieve similar potential clinical benefits.

It was notable that the FPF $(\% \mathrm{ED})$ for SS from Cheng 2 was markedly lower than from Cheng 1, attributable to the higher retention of SS in Cheng 2 compared to Cheng 1 (Tables I and II). In addition, it was obvious that there were differences in the level of IP/PS deposition, MMAD and respirable fractions between the various formulation-device combinations (Table II and Fig. 6). It must be borne in mind that the factors governing deposition within induction ports appear to be drug specific (41), however, it appeared from the current study that the functioning of the reverse-flow cyclone as a spacer depended crucially on the deagglomeration state of the drug particles entering the cyclone. For example, laser diffraction studies (Fig. 7) of the Seretide/Accuhaler ${ }^{\text {TM }}$ formulation revealed the emission of some agglomerates that escaped the cyclone. CFD studies highlighted the potential for vortex instability within the conical region of the spacer which would enable the escape of agglomerates $(27,28)$.

The role of the device in achieving deagglomeration of the formulation prior to entry into the cyclone cannot be ignored when considering the magnitude of the FPF $(\% \mathrm{sED})$ and the IP/PS deposition. FPFs have been demonstrated to be reduced with devices such as the Diskhaler ${ }^{\mathrm{TM}}$ due to the adherence of deagglomerated powder to an impaction grid at the mouthpiece (42). The similar rapid release of a powder plug into the cyclone inlet from the Accuhaler ${ }^{\mathrm{TM}}$ device may not have enabled deagglomeration to be as efficient for SX/FP as for SS from the Cyclohaler®. In the Cyclohaler® a spinning capsule aids powder dispersion and there is also an extended mouthpiece where turbulent flow aids deagglomeration (43). Meanwhile the Easyhaler ${ }^{\text {TM }}$ has a design that maximizes particle separation by changing the dimensions of the airflow (44) prior to emission of the particles from the device.

The differences between the SS fine particle emission of Cheng 1 and Cheng 2 spacers and the reduction of FPD for FP but not SX when the flow rate through the Seretide/ Accuhaler ${ }^{\mathrm{TM}}$ was increased identify a further possible explanation. There is potential that the cyclone does not solely behave as a classifier, but that deagglomeration also takes place within the cyclone vortices. Drug which adheres strongly to the carrier is more difficult to aerosolize than drug which forms agglomerates on the carrier surface (45). Bud (which typically forms agglomerates with lactose) would be more easily removed at equivalent flow rates from the carrier surface than SS (which typically adheres to the surface), thereby leading to higher Bud FPF values. Collisions between particles and the cyclone wall or other particles in the cyclonic flow aids powder dispersal (46), particularly collisions with particles of large mass. Upon increasing the flow rate, sufficient force is provided to break up drug agglomerates further, but also aid removal of surface-adhered drug from the carrier. Thus for example the FPF of SS from Cheng 1 was significantly smaller at $30 \mathrm{Lmin}^{-1}$ than at 45 or $60 \mathrm{Lmin}^{-1}$. In CFD studies $(27,28)$ of cyclone behaviour, it was observed that greater turbulent dispersion forces exist in Cheng 1 owing to its narrower cyclone inlet compared to Cheng 2. The higher force for SS removal from the carrier under the turbulent stress is one possible explanation for the higher FPFs observed with the latter cyclone.

\section{CONCLUSION}

This study has demonstrated the potential to apply miniature reverse-flow cyclones as a generic spacer platform for a range of commercial carrier-based DPIs. The clear benefit in terms of minimizing IP/PS (and hence) oropharyngeal deposition shows the ability to improve the specificity of lung deposition for patients whose inspiratory force is not strong enough to achieve effective formulation deagglomeration and aerosolization. It was also possible to control the aerodynamic particle size distribution of the inhalable aerosol through the device engineering that directs the cut-off diameter of the cyclone. For example using the low cut-off diameters of the cyclones studied herein, it may be possible to extend the ability of marketed DPIs to achieve selective small airway deposition. The study revealed the potential role of deagglomeration 
within the cyclone, and future work will be directed at investigating the deagglomeration mechanisms within miniaturized cyclones, particularly when tested with real inhalation profiles which are achieved by patients through the cyclones.

\section{ACKNOWLEDGMENTS AND DISCLOSURES}

The authors would like to acknowledge the financial support of the University of Hertfordshire and the Engineering Design Centre, University of Cambridge (supported by EPSRC research grant no. EP/E001777/1).

Open Access This article is distributed under the terms of the Creative Commons Attribution License which permits any use, distribution, and reproduction in any medium, provided the original author(s) and the source are credited.

\section{REFERENCES}

1. Lavorini F, Gorrigan C, Barnes P, Dekhuijzen P, Levy M, Pedersen S, et al. Retail sales of inhalation devices in European countries: so much for a global policy. Respir Med. 2011;105(7):1099-103.

2. Broeders MEAC, Sanchis J, Levy ML, Crompton GK, Dekhuijzen PNR, on behalf of the AWG. The ADMIT series-issues in inhalation therapy. 2) Improving technique and clinical effectiveness. Prim Care Respir J. 2009;18(2):76-82.

3. Ganderton D, Kassem NM. Dry powder inhalers. In: Ganderton D, Jones T, editors. Advances in pharamaceutical sciences. London: Academic; 1992. p. 165-91.

4. Srichana T, Martin GP, Marriott C. Dry powder inhalers: the influence of device resistance and powder formulation on drug and lactose deposition in vitro. Eur J Pharm Sci. 1998;7(1):7380 .

5. Hindle M, Byron PR. Dose emissions from marketed dry powder inhalers. Int J Pharm. 1995;116(2):169-77.

6. Chrystyn H. Is inhalation rate important for a dry powder inhaler? using the In-Check Dial to identify these rates. Respir Med. 2003;97(2):181-7.

7. Pauwels R, Newman S, Borgstrom L. Airway deposition and airway effects of antiasthma drugs delivered from metered-dose inhalers. Eur Respir J. 1997;10(9):2127-38.

8. Borgström L, Olsson B, Thorsson L. Degree of throat deposition can explain the variability in lung deposition of inhaled drugs. J Aerosol Med. 2006;19(4):473-83.

9. Islam N, Gladki E. Dry powder inhalers (DPIs) - a review of device reliability and innovation. Int J Pharm. 2008;360(1):1-11.

10. Buhl R. Local oropharyngeal side effects of inhaled corticosteroids in patients with asthma. Allergy. 2006;61(5):518-26.

11. Terzano C. Pressurized metered dose inhalers and add-on devices. Pulmonol Pharmacol Ther. 2001;14(5):351-66.

12. Borgstrom L, Bengtsson T, Derom E, Pauwels R. Variability in lung deposition of inhaled drug, within and between asthmatic patients, with a $\mathrm{PMDI}$ and a dry powder inhaler, Turbuhaler (R). Int J Pharm. 2000;193(2):227-30.

13. Bisgaard H, Pedersen S, Nikander K. Use of budesonide Turbuhaler ${ }^{\circledR}$ in young-children suspected of asthma. Eur Respir J. 1994;7(4):740-2.
14. Clark AR, Hollingworth AM. The relationship between powder inhaler resistance and peak inspiratory conditions in healthy volunteers-implications for in vitro testing. J Aerosol Med. 1993;6(2): 99-110.

15. Kamin WES, Genz T, Roeder S, Scheuch G, Trammer T, Juenemann $\mathrm{R}$, et al. Mass output and particle size distribution of glucocorticosteroids emitted from different inhalation devices depending on various inspiratory parameters. J Aerosol Med Depos Clear Eff Lung. 2002;15(1):65-73.

16. Srichana T, Martin G, Marriott C. A human oral-throat cast integrated with a twin-stage impinger for evaluation of dry powder inhalers. J Pharm Pharmacol. 2000;52(7):771-8.

17. Svartengren K, Lindestad PÅ, Svartengren M, Bylin G, Philipson K, Camner P. Deposition of inhaled particles in the mouth and throat of asthmatic subjects. Eur Respir J. 1994;7(8):1467-73.

18. Al-Showair RAM, Tarsin WY, Assi KH, Pearson SB, Chrystyn H. Can all patients with COPD use the correct inhalation flow with all inhalers and does training help? Respir Med. 2007;101(11):2395-401.

19. DeHaan WH, Finlay WH. In vitro monodisperse aerosol deposition in a mouth and throat with six different inhalation devices. J Aerosol Med. 2001;14(3):361-7.

20. Bisgaard H. Automatic actuation of a dry powder inhaler into a nonelectrostatic spacer. Am J Respir Crit Care Med. 1998;157(2): 518-21.

21. Everard ML, Devadason SG, Le Souef PN. Particle size selection device for use with the Turbohaler. Thorax. 1996;51(5):537-9.

22. Matida EA, Finlay WH, Rimkus M, Grgic B, Lange CF. A new addon spacer design concept for dry-powder inhalers. J Aerosol Sci. 2004;35(7):823-33.

23. Ehtezazi T, Allanson DR, Jenkinson ID, Shrubb I, O'Callaghan C. Investigating improving powder deagglomeration via dry powder inhalers at a low inspiratory flow rate by employing add-on spacers. J Pharm Sci. 2008;97(12):5212-21.

24. Zeng XM, O'Leary D, Phelan M, Jones S, Colledge J. Delivery of salbutamol and of budesonide from a novel multi-dose inhaler Airmax $^{\text {TM }}$. Respir Med. 2002;96(6):404-11.

25. Smith SJ, Harris DS, Striebig RV, Pearl JS. Dry Powder Inhalers. World Patent WO2007 144607 (2007).

26. Needham M, Cocks P, Fradley G. Investigating the efficiency of the $3 \mathrm{M}$ Conix $^{\mathrm{TM}}$ reverse cyclone technology for DPI drug delivery. [Cited 2013 29th April]; Available from: http://solutions.3m.com/ 3MContentRetrievalAPI/BlobServlet?lmd=1320272822000\&locale= en_WW\&asset Type=MMM_Image\&assetId=1319208813657\& blobAttribute=ImageFile.

27. Cheng SJ, Symons DD, editors. Optimisation of dry powder inhalers through robust CFD simulation of air classifiers. Drug Delivery to the Lungs 21; 2010; Edinburgh.

28. Cheng SJ. Numerical and experimental study of cyclone separators for aerosol drug delivery, $\mathrm{PhD}$ Thesis. Cambridge: University of Cambridge; 2012.

29. Hoffmann AC, Stein LE. Gas cyclones and swirl tubes: principles, design and operation. Springer Verlag; 2002.

30. Thiel CG. Cascade impactor data and the lognormal distribution: nonlinear regression for a better fit. J Aerosol Med. 2002;15(4):369-78.

31. Roberts D, Mitchell J. The effect of nonideal cascade impactor stage collection efficiency curves on the interpretation of the size of inhalergenerated aerosols. AAPS Pharm Sci Technol:1-14; 2013.

32. Mitchell JP, Nagel MW, Wiersema KJ, Doyle CG. Aerodynamic particle size analysis of aerosols from pressurized metered-dose inhalers: comparison of Andersen 8-stage cascade impactor, next generation pharmaceutical impactor, and model 3321 aerodynamic particle sizer aerosol spectrometer. AAPS PharmSciTech. 2003;4(4): 425-33.

33. Tarsin WY, Pearson SB, Assi KH, Chrystyn H. Emitted dose estimates from Seretide Diskus and Symbicort Turbuhaler following inhalation by severe asthmatics. Int J Pharm. 2006;316(1-2):131. 
34. Bisgaard H, Klug B, Sumby BS, Burnell PKP. Fine particle mass from the Diskus inhaler and Turbuhaler inhaler in children with asthma. Eur Respir J. 1998;11(5):1111-5.

35. Lavorini F, Fontana GA. Targeting drugs to the airways: the role of spacer devices. Expert Opin Drug Deliv. 2009;6(1):91-102.

36. Barry PW, Ocallaghan C. In vitro comparison of the amount of salbutamol available for inhalation from different formulations used with different spacer devices. Eur Respir J. 1997;10(6):1345-8.

37. Demirkan K, Tolley E, Mastin T, Soberman J, Burbeck J, Self T. Salmeterol administration by metered-dose inhaler alone vs. metereddose inhaler plus valved holding chamber. CHEST J. 2000;117(5): 1314-8.

38. Nair A, Clearie K, Menzies D, Meldrum K, McFarlane L, Lipworth BJ. A novel breath-actuated integrated vortex spacer device increases relative lung bioavailability of fluticasone/salmeterol in combination. Pulmonol Pharmacol Ther. 2009;22(4):305-10.

39. Newman SP, Newhouse MT. Effect of add-on devices for aerosol drug delivery: deposition studies and clinical aspects. J Aerosol Med. $1996 ; 9(1): 55-70$.
40. Donovan MJ, Kim SH, Raman V, Smyth HD. Dry powder inhaler device influence on carrier particle performance. J Pharm Sci; 2012.

41. Tang P, Kwok PCL, Tong Z, Yang R, Raper JA, Chan H-K. Does the United States Pharmacopeia throat introduce de-agglomeration of carrier-free powder from inhalers? Pharm Res:1-11; 2012.

42. Voss A, Finlay WH. Deagglomeration of dry powder pharmaceutical aerosols. Int J Pharm. 2002;248(1):39-50.

43. Daniher DI, Zhu J. Dry powder platform for pulmonary drug delivery. Particuol. 2008;6(4):225-38.

44. Hickey AJ, Crowder TM. Next generation dry powder inhalation delivery systems. Lung Biol Health Dis. 2007;221:445.

45. Begat P, Morton DAV, Staniforth JN, Price R. The cohesive-adhesive balances in dry powder inhaler formulations II: influence on fine particle delivery characteristics. Pharm Res. 2004;21(10):1826-33.

46. Harrison LI, Novak CG, Needham MJ, Ratner P. Comparative pulmonary function and pharmacokinetics of fluticasone propionate and salmeterol xinafoate delivered by two dry powder inhalers to patients with asthma. J Aerosol Med Pulm Drug Del. 2011;24(5): $245-52$ 